\title{
Persistent left superior vena cava: diagnosed by bedside echocardiography in a liver transplant patient
} -a case report-

\author{
Hyerim Kim ${ }^{1}$, Jin Hee Kim², and Hannah Lee \\ Department of Anesthesiology and Pain Medicine, ${ }^{1}$ Seoul National University Hospital, Seoul, ${ }^{2}$ Seoul National University Bundang \\ Hospital, Seongnam, Korea
}

In most cases, persistent left superior vena cava (PLSVC) is asymptomatic and discovered accidentally. This case involves a 43-year-old male who underwent an emergency cadaveric liver transplantation. Postoperatively, the left internal jugular vein was cannulated using a sono-guided Seldinger technique in the intensive care unit. But the chest X-ray showed that the catheter followed the left paramediastinal course instead of crossing midline to the right to enter the superior vena cava. In consideration of the patient's status, an intra-arterial or extra-vascular placement could be excluded. For a diagnosis, we performed a bed-side transthoracic echocardiography with an agitated saline micro-bubble test. When agitated saline was injected through the catheter, the coronary sinus was initially opacified, and then the right atrium followed. In conclusion, we were able to make a diagnosis of PLSVC by a bedside test without radiation exposure. (Korean J Anesthesiol 2014; 67: 429-432)

Key Words: Congenital heart defects, Contrast media, Diagnostic use, Echocardiography.

Persistent left superior vena cava (PLSVC) is not a rare congenital anomaly, but the probability of diagnosis in an actual patient is low [1]. In most cases this anomaly is asymptomatic, and is thereby discovered during central venous catheterization, device implantation, cardiovascular imaging, or surgery [2]. When confronted with an image of a left central venous catheter at a left paramediastinal border, it is important to consider all possibilities, including PLSVC, and to confirm the diagnosis before using or removing it. To our knowledge, there are no case reports in Korea of PLSVC being diagnosed by bedside echocardiography with an agitated saline test in the intensive care unit. Therefore we present a case of PLSVC that was found by an aberrantly positioned central venous catheter on a chest X-ray after liver transplantation.

Received: November 7, 2013. Revised: January 7, 2014. Accepted: January 8, 2014.

Corresponding author: Hannah Lee, M.D., Department of Anesthesiology and Pain Medicine, Seoul National University Hospital, Seoul National University College of Medicine, 101, Daehak-ro, Jongno-gu, Seoul 110-744, Korea. Tel: 82-2-2072-3538, Fax: 82-2-747-5639, E-mail: closerthan@ gmail.com

(c) This is an open-access article distributed under the terms of the Creative Commons Attribution Non-Commercial License (http:// creativecommons.org/licenses/by-nc/3.0/), which permits unrestricted non-commercial use, distribution, and reproduction in any medium, provided the original work is properly cited. 


\section{Case Report}

A 43-year-old male underwent an emergency cadaveric liver transplantation for alcoholic liver cirrhosis. The patient had a history of hypertension and type II diabetes mellitus. His preoperative echocardiogram showed no specific findings. During induction of general anesthesia, arterial cannulation was performed in the right radial artery and right femoral artery. A central venous cannulation was performed with a 9 Fr large bore catheter (Advanced Venous Access, Edwards Lifesciences, Irvine, CA, USA) in the right internal jugular vein using an ultrasound sonographguided Seldinger technique. The estimated blood loss during operation was $2,700 \mathrm{ml}$, and the total amount of infused fluid via the AVA catheter was approximately $4,000 \mathrm{ml}$. The operation was performed without any event, except inserting a left chest tube due to a large amount of pleural effusion.

Postoperatively, the patient was transferred to the surgical intensive care unit. On the second postoperative day we decided to substitute a new central venous catheter for the 9 Fr AVA catheter, which was too large and risky to maintain in the intensive care unit (ICU). The left internal jugular vein was cannulated using a $7 \mathrm{Fr}$ catheter (Arrowgard Blue, Arrow International, Inc., Reading, PA, USA), using an ultrasound sonograph. The catheterization procedure was performed without any difficulty; a J-tip guidewire was inserted without resistance, the blood in the catheter was not pulsatile, and the central venous pressure wave was typical. But the chest X-ray, taken to confirm the position of the central line, showed that the catheter was positioned at the left paramediastinal border instead of crossing the midline to the right to enter the superior vena cava (Fig. 1). The blood

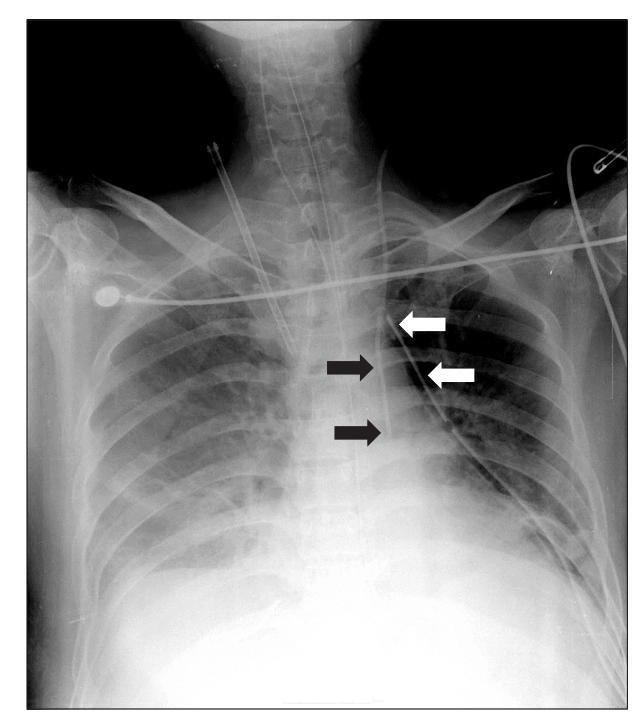

Fig. 1. The left internal jugular catheter (Black arrows) lied along the left paramediastinal border unusually and the chest tube (White arrows) inserted in the left pleural cavity are shown on this radiograph. sample from the catheter looked like venous blood (dark blue rather than bright red). The transduced pressure waveform was the same as the one through the AVA catheter placed at the right internal jugular vein. There was no blood or air leakage through the left-sided chest tube which had been inserted during the operation. A variation of venous anatomy was suggested, but confirmation of the anomaly and exclusion of extra-vascular placement was necessary. A computed tomography or venography with a contrast dye has been the conventional and precise method of investigation. But considering the patient's status, exposure to contrast dye and radiation during the procedure was considered to be harmful. Finally, we performed a bed-side transthoracic echocardiography with an agitated saline test for the diagnosis.

The transthoracic echocardiogram showed a structurally normal heart with a slightly dilated coronary sinus. Air-filled microbubbles were created by shaking $10 \mathrm{ml}$ of saline solution. The agitated saline, used for a contrast agent, was injected through the newly inserted central line catheter. The coronary sinus was opacified with the microbubbles initially, and then the right atrium followed (Fig. 2). This result suggested an anomaly of venous return, persistent left superior vena cava, entering to the right atrium via the coronary sinus. When the agitated saline was injected into the patient's right antecubital vein, the initial opacification was the right atrium providing evidence of a normal right superior vena cava.

Additional echocardiographic examination showed that the aberrantly positioned central venous catheter was neither in an artery nor in the pleural space. Maintenance fluids, intravenous medications, and parenteral nutrition were administered through the left central venous catheter without any problem during ICU stay. Before the patient was transferred to the ward, the central venous catheter was removed safely.

\section{Discussion}

PLSVC is one of the most commonly reported thoracic venous abnormalities. Its incidence is $0.3-0.5 \%$ among healthy populations and $1.5-10 \%$ in patients with congenital abnormalities [1]. PLSVC may present during early fetal life but usually becomes absorbed and attenuated during development. Persistence of the left anterior cardinal vein results in a PLSVC [3]. Approximately $80-92 \%$ of PLSVC drains to the right atrium via the coronary sinus with no hemodynamically significant consequence, and does not cause any symptoms itself. The remainder drains into the left atrium directly or through the pulmonary vein, causing a right to left shunt, and unexplained cyanosis may occur [4].

Although most of the PLSVC cases draining to the right atrium have no physiological derangement, they can cause 

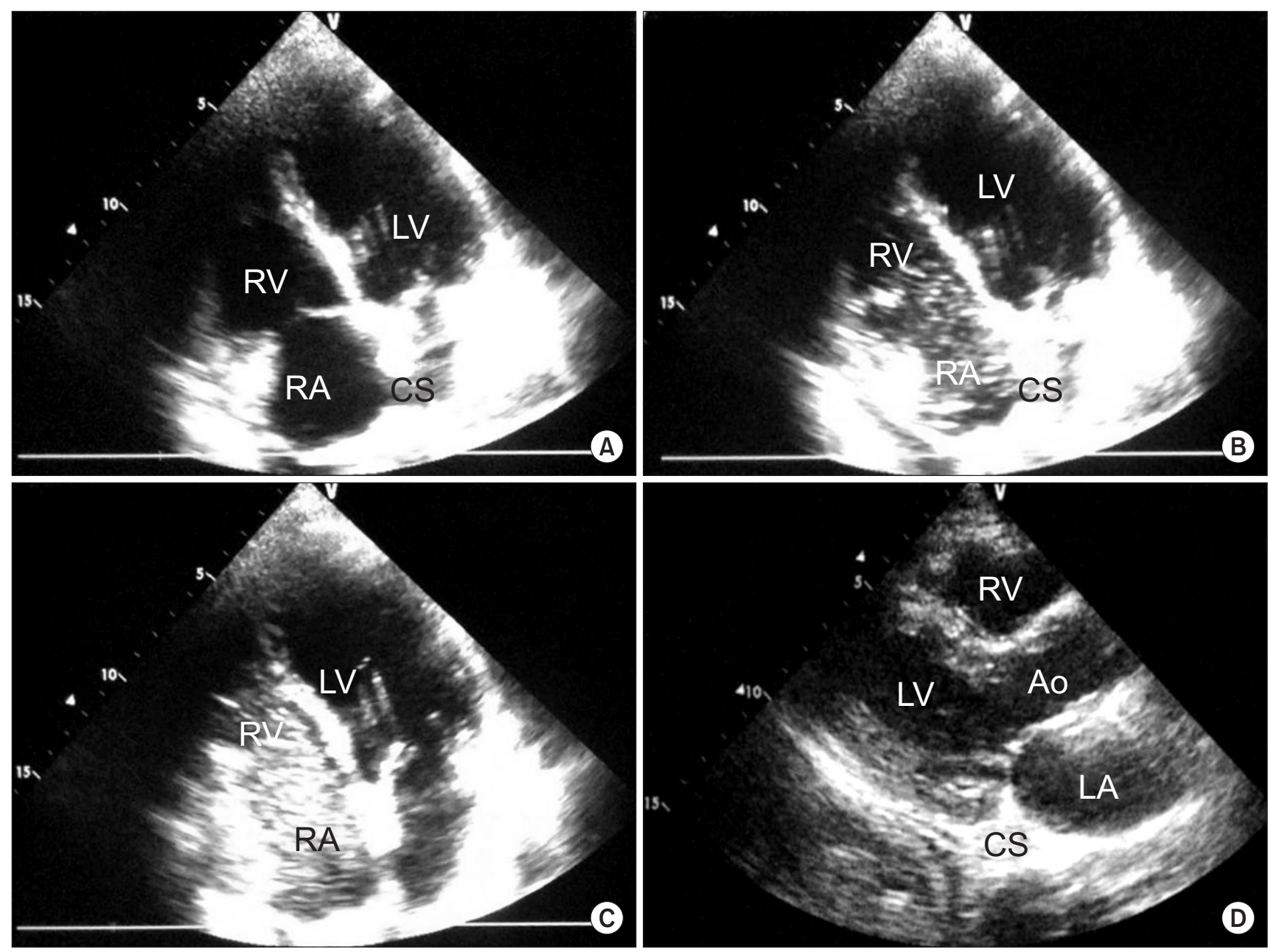

Fig. 2. By injecting agitated saline through the left internal jugular catheter, air-bubble opacification was observed at the coronary sinus (CS), right atrium (RA), and right ventricle (RV), sequentially, in an apical 4-chamber view by transthoracic echocardiography. (A) pre-saline injection, (B) early period after saline injection, (C) late period after saline injection, (D) a dilated coronary sinus by parasternal long-axis view. Ao: aorta, LA: left atrium, LV: left ventricle.

significant clinical consequences in certain situations. When a central line catheter is inserted deeply through a PLSVC and manipulated inside the coronary sinus, it can cause arrhythmia, coronary sinus thrombosis, rupture, cardiac tamponade, and even cardiogenic shock. PLSVC does not allow easy access for Swan-Ganz catheters, permanent pacemakers, or implantable cardioverter defibrillators, which are attributable to orientation when placing through the SVC [5]. Moreover, PLSVC can be a problem during cardiac operation. It causes ineffective venous drainage in cardiopulmonary bypass, and administering retrograde cardioplegics is relatively contraindicated [6].

Patients with PLSVC usually have no known past history related to aberrant veins. Therefore, suspicion is the most important step of diagnosis when the tip of a catheter at the left paramediastinal area is noticed. First, arterial placement is excluded on the basis of blood gas analysis and typical arterial pressure waveform. Clinical signs suggesting PLSVC would be jugular venous distention on the left, and an exaggerated left jugular venous waveform caused by the direct transmission of left atrial contraction [7]. There may be some indications on chest radiographs even though they are not consistent, such as widening of the aortic shadow, a paramedial bulge along the left heart border, or a crescentic vascular shadow projecting from the left upper border of the aortic arch to the middle one-third of the clavicle $[3,4]$. Unfortunately, in this case, we did not find any clinical signs or clues on the chest radiograph, implying PLSVC as discussed above.

When the left internal jugular vein is selected for catheterization, there are other possibilities of venous malposition as well as PLSVC, these include: a left internal thoracic vein [8], a left pericardiophrenic vein [9], a left superior intercostal vein [10], or an accessory hemiazygos vein [11]. Chest pain has been reported 
as the only initial clinical sign of these inadvertent catheter positions, but it is difficult to notice when patients are sedated or on ventilator care in the intensive care unit. The straight descending left-sided projected image of a catheter in an anteroposterior radiograph requires an additional lateral view in order to be distinguished from malpositioning in the left internal mammary vein (behind the sternum), the left pericardiophrenic vein, the PLSVC (middle mediastinum), or the left superior intercostal vein and accessory hemiazygos vein (posterior mediastinum, arching laterally along the aortic arch) [11]. Considering that our patient had liver failure with portal hypertension, portal to systemic collateral circulation would have dilated the tributaries, and thus there was higher risk of malpositioning of the catheter.

The conventional diagnostic tools for PLSVC and for discriminating it from malposition of a catheter are cardiac computed tomography, magnetic resonance imaging, or venography [3]. In spite of their accuracy, these methods are not appropriate for critically ill or hemodynamically unstable patients. Instead, ultrasound echocardiography at bedside is preferred, in regards to some of its beneficial aspects. Echocardiography is preferred over other methods because there is no need for a nephrotoxic contrast agent, no radiation exposure, no risk of transport, its cost-effectiveness, and availability at bed-side [12,13]. An indication for the diagnosis of PLSVC during transthoracic echocardiography is a dilated coronary sinus. Other etiologies having a large coronary sinus are elevated right atrial pressure (most common), coronary arteriovenous fistula, partial anomalous pulmonary venous return, or an unroofed coronary sinus. The subsequent injection of microbubble contrast in left-sided venous catheters is shown firstly in the coronary sinus, and then the right atrium and the right ventricle. On the other hand, when agitated saline is injected into right-sided lines, the right atrium is opacified before the coronary sinus.

If an abnormally positioned central catheter is proved to be due to PLSVC, which drains to the coronary sinus not to the left atrium, the catheter can be left in situ and used as an ordinary central catheter. The central catheter inserted through the PLSVC can be used for large volume access, drug administration, hemodialysis, and so on. However, taking precautions is necessary since the tip could be situated so close to the coronary sinus that it would provoke arrhythmia, thromboembolism, or cardiac tamponade.

In an ICU patient who underwent liver transplantation, the tip of a central catheter was noticed at the left paramediastinal area. An ultrasound echocardiograph with agitated saline was enabled bedside the diagnosis of PLSVC and avoided radiation exposure.

\section{References}

1. Biffi M, Boriani G, Frabetti L, Bronzetti G, Branzi A. Left superior vena cava persistence in patients undergoing pacemaker or cardioverterdefibrillator implantation: a 10-year experience. Chest 2001; 120: 139-44.

2. Dearstine M, Taylor W, Kerut EK. Persistent left superior vena cava: chest x-ray and echocardiographic findings. Echocardiography 2000; 17: 453-5.

3. Lönnqvist PA, Olsson GL. Persistent left superior vena cava--an unusual location of central venous catheters in children. Intensive Care Med 1991; 17: 497-500.

4. Higgs AG, Paris S, Potter F. Discovery of left-sided superior vena cava during central venous catheterization. Br J Anaesth 1998; 81: $260-1$.

5. Peltier J, Destrieux C, Desme J, Renard C, Remond A, Velut S. The persistent left superior vena cava: anatomical study, pathogenesis and clinical considerations. Surg Radiol Anat 2006; 28: 206-10.

6. Shahian DM. Retrograde coronary sinus cardioplegia in the presence of persistent left superior vena cava. Ann Thorac Surg 1992; 54: 1214-5.

7. Spearman P, Leier CV. Persistent left superior vena cava: unusual wave contour of left jugular vein as the presenting feature. Am Heart J 1990; 120: 999-1002.

8. Liao HR, Li TS, Chen KB, Li CY, Jeng LB, Yang HR, et al. Rare malposition of a central venous catheter into the left internal mammary vein in a liver transplantation patient. J Anesth 2011;25: 462-4.

9. Zaman MH, Mitra P, Bondi E, Gintautas J, Abadir AR. A rare malposition of the central venous catheter. Chest 1990; 98: 768-70.

10. Padovan RS, Paar MH, Aurer I. (Mis)placed central venous catheter in the left superior intercostal vein. Radiol Oncol 2011; 45: 27-30.

11. Muhm M, Sunder-Plassmann G, Druml W. Malposition of a dialysis catheter in the accessory hemiazygos vein. Anesth Analg 1996; 83: 883-5.

12. Walpot J, Pasteuning WH, van Zwienen J. Persistent left superior vena cava diagnosed by bedside echocardiography. J Emerg Med 2010; 38 : 638-41.

13. Kurtoglua E, Cakinb O, Akcayc S, Akturkd E, Korkmaza H. Persistent left superior vena cava draining into the coronary sinus. Cardiol Res 2011; 2: 249-52. 